\title{
Clinico-Pathological Characterization of Hereditary, Familial and Sporadic Prostate Cancer
}

\author{
Diem Nguyen Bentzon, Anne Sofie Lynnerup, Michael Borre \\ Department of Urology, Aarhus University Hospital Skejby, Aarhus, Denmark \\ Email: diem.bentzon@dadlnet.dk
}

Received January 12, 2012; revised February 7, 2012; accepted February 28, 2012

\begin{abstract}
Aim: To characterize familial prostate cancer including hereditary prostate cancer and assess the disease-free survival following radical prostatectomy. Methods: A self-administered written questionnaire was forwarded to 709 prostatectomized patients from the Aarhus Prostate Cancer Study containing questions about cases of prostate cancer (PC), age at diagnosis, vital status, and age at death for all first-degree relatives. Patients were then divided into groups according to their family history: hereditary prostate cancer (HPC), familial prostate cancer (FPC), and sporadic prostate cancer (SPC) groups. The information from a subset of both FPC $(n=17)$ and SPC $(n=17)$ groups were validated in the Danish Cancer Register and the Civil Registration System. Between groups, we described the association of age, prostatespecific antigen (PSA), postoperative Gleason score and T Stage. A Kaplan-Meier curve demonstrated postoperative disease-free survival in each group. Results: The response rate was $81 \%(574 / 709)$. About $21 \%$ of the patients were categorized in the FPC group, of which 7\% were identified as having HPC. The median follow-up time was 63 months for HPC, 65 months for FPC and 88 months for SPC. Overall, there was no significant difference between groups in clinical features and disease-free survival except that patients with HPC were significantly associated with younger age than sporadic cases $(p=0.02)$. The proportion of self-reported PC diagnoses confirmed in the cancer register was $27.8 \%$. The index persons with SPC reported no PC in first-degree relatives and none was found the cancer register. Conclusion: Overall, we found no difference in clinical characteristics and survival, following radical prostatectomy except that patients with HPC were younger at diagnosis. These results are in line with previously reported data.
\end{abstract}

Keywords: Descriptive Epidemiology; Family History; Self-Reports; Clinico-Pathology

\section{Introduction}

In 2008, prostate cancer (PC) was the second most frequently diagnosed cancer disease in men worldwide with an incidence of 913,000 cases [1,2]. Approximately 4000 new cases were diagnosed in Denmark in 2009 making $\mathrm{PC}$ the most common cancer type in men [3]. PC is a heterogeneous cancer disease and at present, it is difficult to foresee which PC cases are going to progress and which are not. It is important to find ways of distinguishing between indolent and aggressive forms to avoid unnecessary prostatectomy and related morbidities. Family history is one of the strongest risk factors for PC. Having a first-degree relative with $\mathrm{PC}$ increases the risk of developing the disease by 2.5 -fold, and the risk increases additionally if the relative has early-onset of PC or if multiple first-degree relatives are diagnosed with PC [4]. Family history and twin studies support the notion that heredity plays an important role in PC, and observations of clustering in certain families gave rise to the definition of hereditary prostate cancer (HPC) [5-8] HPC does not seem to differ clinico-pathologically from the sporadic form except that disease presentation has been reported to be $6-7$ years earlier $[5,9,10]$.

Despite several large epidemiological studies, there is not yet clear evidence for clinico-pathological characteristics that can be used to guide decisions about which PC patients need treatment. Patients who have undergone radical prostatectomy for clinically localized PC (cT1ccT2c) are described clinically when enrolled in the Aarhus Prostate Cancer Study. The aim of this study was to identify and characterize individuals with an inherited susceptibility to PC and investigate the impact of family history on the prognosis.

\section{Methods and Materials}

\subsection{Aarhus Prostate Cancer Study}

This study was based on data from the Aarhus Prostate Cancer Study. Since 1997, eligible individuals have been enrolled consecutively into the Aarhus Prostate Cancer Study if diagnosed with PC at the Department of Urology, Aarhus University Hospital. All data have been prospec- 
tively collected including clinical and biochemical data on all patients undergoing radical prostatectomy between April 1997 and April 2010. Every visit at the outpatient clinic has been registered, both before and after surgery. Patients have been followed at the outpatient clinic at standard intervals: 3 months, 6 months and 12 months after surgery. Subsequently, the patients' general practitioners have performed annual clinical and biochemical follow-up. The results of tests and examinations relevant to the study performed at the patient's referring hospital have been retrieved together with the medical records.

The Danish Data Protection Agency approved the study. Written and informed consent was obtained from all participants.

The study population comprised 731 consecutive patients from the Aarhus Prostate Cancer Study that were diagnosed with clinically localized PC and underwent radical prostatectomy from 1997 to 2010 at Aarhus University Hospital (Figure 1). Of the 731 individuals, 22 patients were excluded for various reasons (i.e. unknown addresses, deaths, adoption, and dropouts). Between September 2007 and November 2009, a self-administered written questionnaire was therefore forwarded to 709 enrolled patients. The completion of the questionnaire was

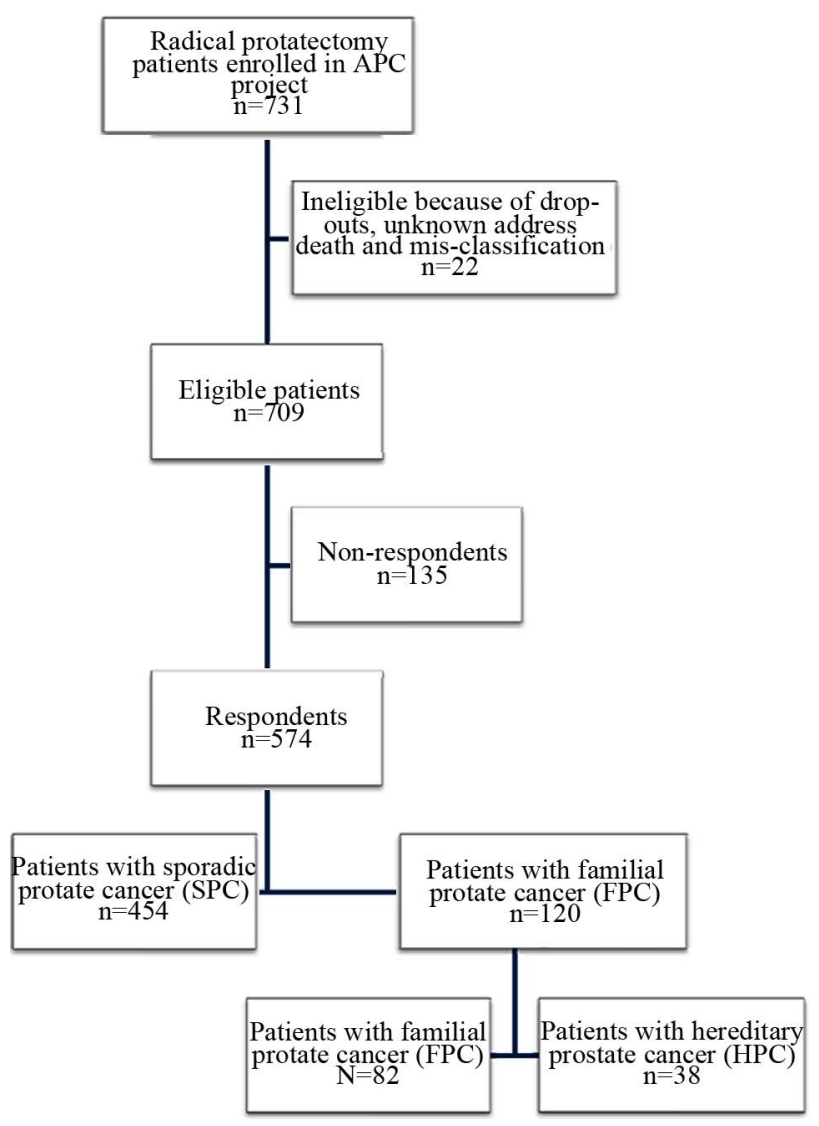

Figure 1. Eligibility and recruitment procedure of the participants. APC Study: Aarhus Prostate Cancer Study. ensured by a reminder letter containing another copy of the questionnaire, but no more than three reminders were permitted. The relationship to the respondent, age at diagnosis, vital status, or age at death were requested for all first-degree relatives. All questionnaires were evaluated and used to divide respondents into two groups: 1) patients with familial prostate cancer (FPC); and 2) participants with sporadic prostate cancer (SPC). The FPC group was defined by at least two first-degree relatives with PC, including the index person, and included cases with HPC identified according to the eligibility criteria by Carter et al. [5]. The Carter criteria were 1) at least three first-degree relatives diagnosed with PC; 2) two first-degree relatives with PC diagnosed before age 56; or 3) at least one PC case in each generation, either paternal or maternal lineage, through three generations. The SPC group included cases with no reported family history of PC.

\subsection{Identification of Relatives and Cancer Diagnosis Verification}

All Danish citizens, living or born on 1 April 1968, have been given a unique personal identification number. This identification number secures linkage between all Registries in the health and social systems. Verification of selfreports was attempted for a selected subset of 17 patients with a family history of PC and an age-matched group of 17 SPC cases. From the questionnaires, the names and date of birth of the first-degree relatives were obtained and these were confirmed in the Civil Registration System. This system does not contain links between family members before 1960; however, our entire study population was born before 1960. If no information could be obtained on first-degree relatives, the Danish State Archive in the region where the family member was born was contacted. The Danish State Archive was established in 1891 and has continuously collected information from church books and, since 1924, from the National Registration Office. If the Civil Registration System or the States Archives could not provide the data of the relatives, the National Registration Office in the municipality where the family member was born was contacted. Every first-degree relative identified through these searches were once more validated in the Civil Registration System and then linked to the Danish Cancer Registry using their personal identification number or by their full name and date of birth. The Danish Cancer Registry was established in 1942 and is based on compulsory reports of diagnosed cancers, with a coverage close to $100 \%[11$, 12]. All cancer diagnoses including $P C$ and any other cancers, diagnosed before November 2009, were identified.

\subsection{Statistical Analyses}

Data management and statistical analyses were performed 
using Microsoft Office Excel 2007 (Microsoft Corp, United States) and Intercooled Stata 10.0 (StataCorp LP, Texas, USA). Frequency distributions for survey variables by groups and overall were generated to describe the study sample. Preoperative variables including PSA, Gleason score and $\mathrm{T}$ stage of prostate tumors were classified according to the D'Amico clinical risk score [13]. Age was a normally distributed variable and the means were compared between FPC, HPC and SPC using ttest, equal variances assumed (standard deviation test). The median of PSA was calculated and the difference between the groups was compared by using the Mann-Whitney test. Using Pearson's chi-square and Fisher's exact test, the categorical parameters were assessed, including postoperative Gleason score ( $\leq 6$ vs. 7 vs. $\geq 8$ ) and postoperative T stage ( $\leq$ pT2a vs. pT2b vs. $\geq p T 2 c)$. Using the Kaplan Meier method, we performed disease-free survival curve and log rank test for statistical differences between groups. Time at risk was calculated from the date of surgery to the date of biochemical recurrence or PC-specific death; other patients were censored at their last blood test. Biochemical relapse was determined by one single PSA measurement $\geq 0.2 \mathrm{ng} / \mathrm{ml}$ after radical prostatectomy. The estimates were given with $95 \%$ confidence interval $(95 \%$ $\mathrm{CI}$ ), and significance was achieved at $p<0.05$.

\section{Results}

We sent out Questionnaires were sent to 709 eligible individuals and we received answers from 574 (80.9\%) respondents. Of the 574 respondents, 120 (20.9\%) stated a family history with at least two cases of $\mathrm{PC}$ in the family and were hence assigned to the FPC group. Of those, $38(6.6 \%)$ cases also fulfilled the definition of HPC according to Carter et al.'s criteria [5]. The remaining 454 (79.1\%) men were included in the SPC group. Generally, the patients (index persons) could not account for when their relatives (probands) had been diagnosed with PC and they tended to provide information only about those who had a PC diagnosis. Validation of PC diagnoses was conducted for 17 index persons with FPC as well as for 17 men with SPC. However, only first-degree relatives were validated, a total of 228 first-degree relatives were identified and confirmed in the Civil Registration System, and they were almost equally distributed between men (n $=109)$ and women $(\mathrm{n}=119)$. Of 228 first-degree relatives, 113 were relatives to the FPC cases and 115 were relatives to the SPC cases. During the investigation, 144 of 228 relatives were identified although they were not mentioned in the questionnaires. Index persons with FPC had 18 family members diagnosed with PC, mainly fathers and brothers, of which 5 were confirmed in the Danish Cancer Registry. The proportion of self-reported PC confirmed in the register was $27.8 \%(5 / 18)$. The Cancer Register confirmed that index persons with SPC had no relatives diagnosed with PC. After validation, 12 index persons from the FPC group were included in the SPC group.

Demographic information and clinical characteristics including age at diagnosis, family history of PC and any cancer diagnoses in first-degree relatives, pre- and postoperative PSA, Gleason score and Tumor Node Metastasis stage (TNM) are summarized in Table 1. Kaplan-Meier curves for biochemical relapse-free survival are shown in Figure 2. The median follow-up time was 65 months for the FPC group, 88 months for the HPC group and 63 months for SPC group. The mean age at diagnosis was 62.2 years in patients with FPC, 60.9 years in patients with HPC and 63.1 in patients with SPC. Overall, there were no significant differences except for HPC patients were significantly younger than SPC patients $(p=0.02)$.

\section{Discussion}

Family history is one of the strongest risk factors for PC. Many large cohort studies have consistently reported a marked clustering of PC in some families with PC risk associated with the age at diagnosis of the kinship and the number of relatives $[4,5,14]$. The definition of HPC is currently based on epidemiological observations, and there are no reported clinical traits that clearly separate HPC from SPC. In this study, we assessed the clinical and pathological characteristics of FPC, HPC and SPC as well as disease-free survival. We found that there were no significant differences between FPC, HPC and SPC cases except that patients with HPC were significantly younger than patients with SPC. These findings are in accordance with previous studies.

In this study, the FPC accounted for $20.9 \%$ of all PC cases and HPC for $6.6 \%$; this was in line with previously reported data $[15,16]$.

The patients were diagnosed from 1998 and onwards. After radical prostatectomy, the patients entered the control programme for $6-12$ months at the outpatient clinic, including regular PSA assessment. Hereafter, they were discharged to be followed at the general practitioner. The short median follow-up time (two to two and a half years) reflected, among others, the misconception among many patients that PSA assessment on a regular basis after surgery was not necessary. The survival curves for FPC and SPC were parallel until 60 months after surgery where the survival of FPC cases became worse than SPC (Figure 1). The disease-free survival of HPC and SPC had a similar course.

There were no significant differences between HPC, FPC, and SPC in tumor variables and biochemical recurrence (Table 1). These findings are in agreement with previous reports; Gronberg et al. detected no significant difference in survival or tumor grade between cohorts of 249 SPC, 46 HPC and 258 FPC cases [17]. Valeri et al. 
Table 1. Clinico-pathological characteristics of cases of sporadic (SPC), familial (FPC) and hereditary (HPC) prostate cancer. a $p$-values for comparisons between FPC and SPC; ${ }^{a} p$-value for comparisons between HPC and SPC; "ttest; " $l o g$ rank test; ${ }^{\S}$ Mann-Whitney; and ${ }^{\circledR}$ Pearson's chi-square test or Fisher's exact test.

\begin{tabular}{|c|c|c|c|c|c|}
\hline & FPC & $p$-value ${ }^{\mathrm{a}}$ & SPC & $p$-value ${ }^{\text {b }}$ & HPC \\
\hline \multicolumn{6}{|l|}{ Characteristics } \\
\hline Number (\%) & $120(20.9)$ & & $454(79.1)$ & & $38(6.6)$ \\
\hline \multicolumn{6}{|l|}{ Age at diagnosis - yrs } \\
\hline Mean $(95 \% \mathrm{CI})$ & $62.2(62.2-63.2)$ & $0.10^{*}$ & $63.1(62.6-63.6)$ & $0.02^{*}$ & $60.9(59.2-62.6)$ \\
\hline \multicolumn{6}{|l|}{ First-degree relatives-no. (\%) } \\
\hline 0 relative & $0(0.0)$ & & $454(100.0)$ & & $0(0.0)$ \\
\hline 1 relatives & $82(68.3)$ & & $0(0.0)$ & & $0(0.0)$ \\
\hline 2 relatives & $24(20.0)$ & & $0(0.0)$ & & $24(63.2)$ \\
\hline$\geq 3$ relatives & $14(11.7)$ & & $0(0.0)$ & & $14(36.8)$ \\
\hline Biochemical failure & & $0.22^{\#}$ & & $0.50^{\#}$ & \\
\hline Follow up (months) - median & 23 & & 24.3 & & 30.2 \\
\hline \multirow{3}{*}{$\begin{array}{l}\text { Recurrence } \\
\text { D'Amico score }\end{array}$} & $77(64.2)$ & & $310(68.3)$ & & $22(57.9)$ \\
\hline & $43(35.8)$ & & $144(31.7)$ & & $16(42.1)$ \\
\hline & \\
\hline Low & $17(14.2)$ & & $60(13.2)$ & & $7(18.4)$ \\
\hline Intermediate & $43(35.8)$ & & $172(37.9)$ & & $15(39.5)$ \\
\hline High & $60(50.0)$ & & $222(48.9)$ & & $16(42.1)$ \\
\hline \multicolumn{6}{|l|}{ PSA } \\
\hline Median & 65 & $0.24^{\S}$ & 88 & $0.89^{\S}$ & 63 \\
\hline Gleason score-no. (\%) & & $0.50^{\circledR}$ & & $0.47^{\circledR}$ & \\
\hline$\leq 6$ & $52(43.3)$ & & $223(49.1)$ & & $19(50.0)$ \\
\hline 7 & $58(48.3)$ & & $197(43.4)$ & & $17(44.7)$ \\
\hline$\geq 8$ & $7(5.8)$ & & $29(5.3)$ & & $2(5.3)$ \\
\hline Missings & $3(2.5)$ & & $5(0.0)$ & & $0(0.0)$ \\
\hline Tumor stage - no. $(\%)$ & & $0.33^{\circledR}$ & & $0.39^{\circledR}$ & \\
\hline$\leq \mathrm{pT} 2 \mathrm{a}$ & $13(10.8)$ & & $61(13.4)$ & & $4(10.5)$ \\
\hline pT2b & $3(2.5)$ & & $17(3.7)$ & & $0(0.0)$ \\
\hline$\geq \mathrm{pT} 2 \mathrm{c}$ & $103(85.8)$ & & $374(82.4)$ & & $34(89.5)$ \\
\hline $\mathrm{T}_{\mathrm{x}}$ & $1(0.8)$ & & $2(0.4)$ & & $0(0.0)$ \\
\hline \multicolumn{6}{|l|}{ Nodal stage - no. $(\%)$} \\
\hline $\mathrm{N}_{0}$ & $77(64.2)$ & & $336(74.0)$ & & $26(68.4)$ \\
\hline $\mathrm{N}_{1}$ & $1(0.8)$ & & $7(1.5)$ & & $1(2.6)$ \\
\hline $\mathrm{N}_{\mathrm{x}}$ & $42(35.0)$ & & $111(24.2)$ & & $11(28.9)$ \\
\hline \multicolumn{6}{|l|}{ Metastatis stage - no. $(\%)$} \\
\hline $\mathrm{M}_{0}$ & $112(93.3)$ & & $427(94.1)$ & & $36(94.7)$ \\
\hline $\mathrm{M}_{1}$ & $0(0.0)$ & & $0(0.0)$ & & $0(0.0)$ \\
\hline $\mathrm{M}_{\mathrm{x}}$ & $8(6.7)$ & & $27(5.9)$ & & $2(5.9)$ \\
\hline
\end{tabular}

investigated diagnostic modalities, age, pathological tumor grading and staging, and PSA in cases with FPC, HPC, and SPC [18]. Age was the only significant parameter differing between groups; HPC was detected 4.7 years earlier than sporadic cases $(p<0001)$. Similarly, in a study population of men undergoing radical prostatectomy, Sacco et al. found that the HPC group was five years younger than the SPC group $(p<0001)$, otherwise, no significant differences were observed in clinical and pathological variables $[16,19]$.

Younger age at diagnosis among cases with family history is expected for a genetically determined disease. However, early diagnosis of PC may in part be explained by an increased awareness among yet unaffected members in HPC families once a close relative is diagnosed with PC. The fact that the difference in age at diagnosis between HPC and SPC was smaller in the present study compared to that reported in other studies may be because PSA screening is not yet implemented in Denmark although it is much debated. Furthermore, the PC awareness has been increased among urologists only in the last or two decades as more treatment modalities including radical surgery and radiation therapy were introduced.

HPC is much more complex than initially anticipated. The genetic heterogeneity of HPC may underlie the conflicting reports of the clinico-pathological presentation of 

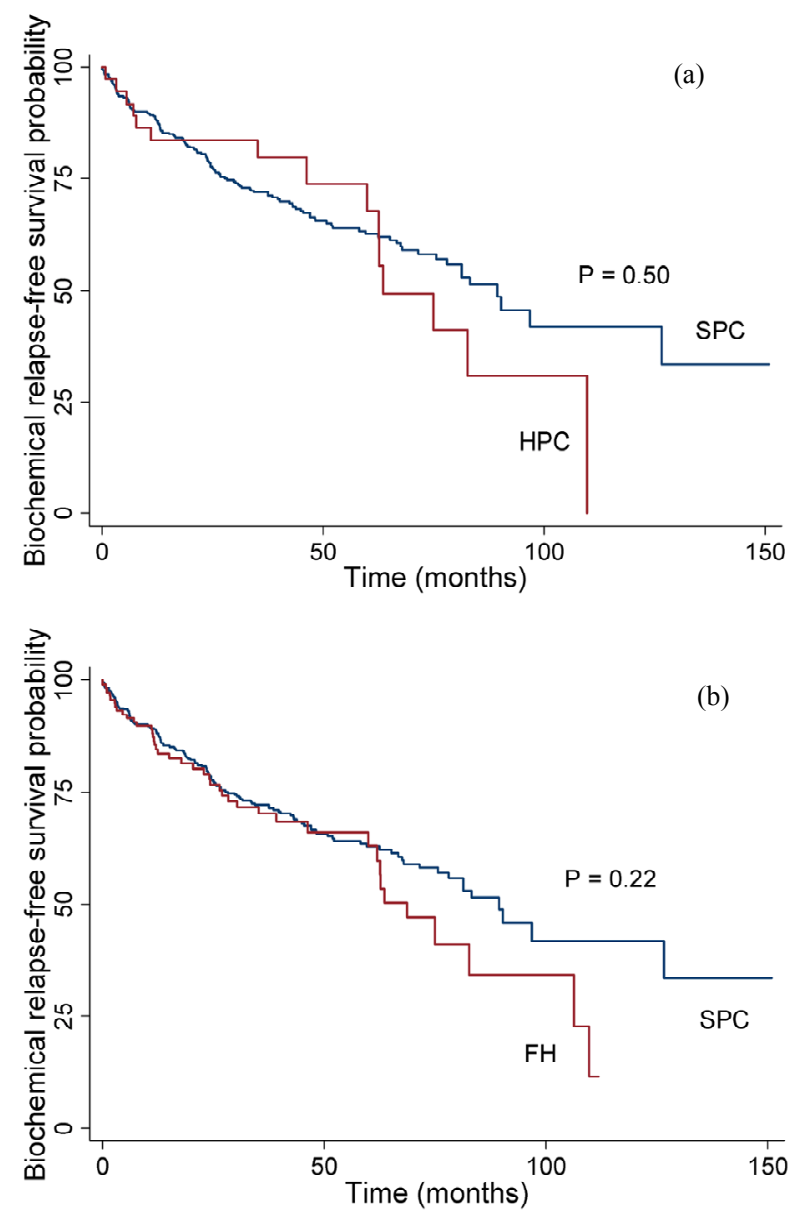

Figure 2. Biochemical relapse-free survival probability for patients with HPC (a) and FPC (b) compared with SPC cases.

the disease. Some reported HPC being associated with low grade and or localized tumor [18,20,21]. In other studies, prostate cancer in families linked to $H P C 1$ was characterized by early onset and high grade or stage [22-24]. Disease outcome were investigated as well. In a study, patients with a family history of PC were associated with biochemical recurrence following radical prostatectomy [25]. In other studies, however, relapse-free survival did not differ between SPC and HPC or patients with a family history $[10,26]$. Thus, the clinical and pathological characteristics of HPC remain controversial at all clinical stages $[10,18,27]$.

Although HPC is a genetically heterogeneous cancer disease, it displays noteworthily scarce differences in clinical characteristics between HPC and SPC. Without specific hereditary prostate cancer genes and with present operational definition of HPC based on epidemiological criteria, it is difficult to discriminate true hereditary cases from non-hereditary cases and HPC families may include members with likely "phenocopies" [5].
We validated the self-reports of PC in first-degree relatives using the Civil Registration System and the Danish Cancer Registry. Seventeen FPC cases were randomly selected and 17 SPC cases were age-matched. Contrary to what we would expect, the concordance between selfreports and the Danish Cancer Registry was poor for the HPC cases. It was stated that 18 first-degree relatives (nine fathers and nine brothers) were diagnosed with PC; however, only five (one father and four brothers) of those were confirmed by the cancer register. The cancer Register confirmed that first-degree relatives of SPC cases were not diagnosed with PC. Several population-based studies addressing the accuracy of self-reports observed both high and low accuracy rates for PC; however, the inconsistency may in part be due to cultural factors e.g. PC awareness and treatment modalities [28-30]. The patients with a family history were all in the 70's except for one case (51 years) and their brothers likewise. Their fathers died in the early 80 s, which is prior to the introduction of PSA assessment and treatment modalities in Denmark. Although the fathers had PC, the diagnosis would not be found in any medical records. This may explain the discrepancy between the cancer register and the self-reported PC diagnoses in fathers; however, this explanation cannot justify the discrepancy regarding the brothers. When reporting PC in brothers (and fathers), the patients with family history might misinterpret any symptom in the prostate as PC.

Potential limitations of our study are misclassification; the proportion of true hereditary cases is unknown as long as the HPC definition is based on family history. Moreover, $\mathrm{PC}$ awareness in certain families may increase the number of medical contacts, and PC may therefore be detected earlier in those families because of a strong family history. Patients included in this study all underwent radical prostatectomy and they might not be representative for the whole spectrum of PC as patients with very aggressive $\mathrm{PC}$ that had a poor prognosis and were likely to die from PC at a young age were not included. The last issues to be addressed are the small sample size, in particular the HPC group, and the short follow-up time.

\section{Conclusion}

No differences were observed in disease-free survival, tumor staging and grading between HPC, FPC, and SPC groups. Our data confirmed results of previous studies that patients with HPC or a family history were signifycantly younger than sporadic cases. These results suggest that patients with HPC should not be treated differently from patients with SPC.

\section{Perspectives}

Future studies may focus on the molecular and genetic 
characterization of the study samples. However, in future studies, we intend to include advanced PC cases that are likely to progress rapidly as the current cohort comprises only patients with localized PC.

\section{REFERENCES}

[1] J. Ferlay, H. R. Shin, F. Bray, D. Forman, C. Mathers and D. M. Parkin, "Estimates of Worldwide Burden of Cancer in 2008: GLOBOCAN 2008," International Journal of Cancer, Vol. 127, No. 12, 2010, pp. 2893-2917. doi:10.1002/ijc. 25516

[2] A. Jemal, M. M. Center, C. DeSantis and E. M. Ward, "Global Patterns of Cancer Incidence and Mortality Rates and Trends," Cancer Epidemiology, Biomarkers \& Prevention, Vol. 19, 2010, pp. 1893-1907.

doi:10.1158/1055-9965.EPI-10-0437

[3] Sundhedsstyrelsen, "Cancerregisteret," 2009.

[4] M. P. Zeegers, A. Jellema and H. Ostrer, "Empiric Risk of Prostate Carcinoma for Relatives of Patients with Prostate Carcinoma: A Meta-Analysis," Cancer, Vol. 97, No. 8, 2003, pp. 1894-1903. doi:10.1002/cncr.11262

[5] B. S. Carter, G. S. Bova, T. H. Beaty, et al., "Hereditary Prostate Cancer: Epidemiologic and Clinical Features," The Journal of Urology, Vol. 150, No. 3, 1993, pp. 797-802.

[6] W. F. Page, M. M. Braun, A. W. Partin, N. Caporaso and P. Walsh, "Heredity and Prostate Cancer: A Study of World War II Veteran Twins," The Prostate, Vol. 33, No. 4, 1997, pp. 240-245. doi:10.1002/(SICI)1097-0045(19971201)33:4<240::AIDPROS3>3.0.CO;2-L

[7] P. Lichtenstein, N. V. Holm, P. K. Verkasalo, et al., "Environmental and Heritable Factors in the Causation of Cancer-Analyses of Cohorts of Twins from Sweden, Denmark, and Finland," The New England Journal of Medicine, Vol. 343, No. 2, 2000, pp. 78-85. doi:10.1056/NEJM200007133430201

[8] S. G. Baker and B. S. Kramer, "Using Microarrays to Study the Microenvironment in Tumor Biology: The Crucial Role of Statistics," Seminars in Cancer Biology, Vol. 18, No. 5, 2008, pp. 305-310. doi:10.1016/j.semcancer.2008.03.001

[9] O. Bratt, U. Kristoffersson, H. Olsson and R. Lundgren, "Clinical Course of Early Onset Prostate Cancer with Special Reference to Family History as a Prognostic Factor," European Urology, Vol. 34, No. 1, 1998, pp. 19-24.

[10] O. Bratt, J. E. Damber, M. Emanuelsson and H. Gronberg, "Hereditary Prostate Cancer: Clinical Characteristics and Survival," The Journal of Urology, Vol. 167, No. 6, 2002, pp. 2423-2426. doi:10.1016/S0022-5347(05)64997-X

[11] H. H. Storm, "Completeness of Cancer Registration in Denmark 1943-1966 and Efficacy of Record Linkage Procedures," International Journal of Epidemiology, Vol. 17, No. 1, 1988, pp. 44-49. doi:10.1093/ije/17.1.44

[12] Osterlind and O. M. Jensen, "Evaluation of Cancer Registration in Denmark in 1977. Preliminary Evaluation of Cancer Registration by the Cancer Register and the National Patient Register," Ugeskrift for Laeger, Vol. 147, No.
31, 1985, pp. 2483-2488.

[13] V. D'Amico, R. Whittington, S. B. Malkowicz, et al., "Biochemical Outcome after Radical Prostatectomy, External Beam Radiation Therapy, or Interstitial Radiation Therapy for Clinically Localized Prostate Cancer," The Journal of the American Medical Association, Vol. 280, No. 11, 1998, pp. 969-974. doi:10.1001/jama.280.11.969

[14] Y. C. Chen, J. H. Page, R. Chen and E. Giovannucci, "Family History of Prostate and Breast Cancer and the Risk of Prostate Cancer in the PSA Era," The Prostate, Vol. 68, No. 14, 2008, pp. 1582-1591. doi:10.1002/pros.20825

[15] B. S. Carter, T. H. Beaty, G. D. Steinberg, B. Childs and P. C. Walsh, "Mendelian Inheritance of Familial Prostate Cancer," Proceedings of the National Academy of Sciences of the United States of America, Vol. 89, No. 8, 1992, pp. 3367-3371. doi:10.1073/pnas.89.8.3367

[16] E. Sacco, T. Prayer-Galetti, F. Pinto, et al., "Familial and Hereditary Prostate Cancer by Definition in an Italian Surgical Series: Clinical Features and Outcome," European Urology, Vol. 47, No. 6, 2005, pp. 761-768. doi:10.1016/j.eururo.2005.01.016

[17] H. Gronberg, L. Damber, B. Tavelin and J. E. Damber, "No Difference in Survival between Sporadic, Familial and Hereditary Prostate Cancer," British Journal of Urology, Vol. 82, No. 4, 1998, pp. 564-567. doi:10.1046/j.1464-410X.1998.00801.x

[18] A. Valeri, R.Azzouzi, E. Drelon, et al., "Early-Onset Hereditary Prostate Cancer Is Not Associated with Specific Clinical and Biological Features," The Prostate, Vol. 45, No. 1, 2000, pp. 66-71. doi:10.1002/1097-0045(20000915)45:1<66::AID-PROS8 3.0.CO;2-W

[19] K. A. Roehl, S. Loeb, J. A. Antenor, N. Corbin and W. J. Catalona, "Characteristics of Patients with Familial versus Sporadic Prostate Cancer," The Journal of Urology, Vol. 176, No. 6, 2006, pp. 2438-2342. doi:10.1016/j.juro.2006.07.159

[20] D. W. Keetch, P. A. Humphrey, D. S. Smith, D. Stahl and W. J. Catalona, "Clinical and Pathological Features of Hereditary Prostate Cancer," The Journal of Urology, Vol. 155, No. 6, 1996, pp. 1841-1843. doi:10.1016/S0022-5347(01)66024-5

[21] S. I. Bastacky, K. J. Wojno, P. C. Walsh, M. J. Carmichael and J. I. Epstein, "Pathological Features of Hereditary Prostate Cancer," The Journal of Urology, Vol. 153, No. 3, 1995, pp. 987-992. doi:10.1016/S0022-5347(01)67619-5

[22] H. Gronberg, J. Xu, J. R. Smith, et al., "Early Age at Diagnosis in Families Providing Evidence of Linkage to the Hereditary Prostate Cancer Locus (HPC1) on Chromosome 1," Cancer Research, Vol. 57, No. 21, 1997, pp. 4707-4709.

[23] H. Gronberg, S. D. Isaacs, J. R. Smith, et al., "Characteristics of Prostate Cancer in Families Potentially Linked to the Hereditary Prostate Cancer 1 (HPC1) Locus," The Journal of the American Medical Association, Vol. 278, No. 15, 1997, pp. 1251-1255. doi:10.1001/jama.1997.03550150055035

[24] E. L. Goode, J. L. Stanford, M. A. Peters, et al., "Clinical 
Characteristics of Prostate Cancer in an Analysis of Linkage to Four Putative Susceptibility Loci," Clinical Cancer Research, Vol. 7, No. 9, 2001, pp. 2739-2749

[25] P. A. Kupelian, V. A. Kupelian, J. S. Witte, R. Macklis and E. A. Klein, "Family History of Prostate Cancer in Patients with Localized Prostate Cancer: An Independent Predictor of Treatment Outcome," Journal of Clinical Oncology, Vol. 15, No. 4, 1997, pp. 1478-1480.

[26] J. J. Bauer, S. Srivastava, R. R. Connelly, et al., "Significance of Familial History of Prostate Cancer to Traditional Prognostic Variables, Genetic Biomarkers, and Recurrence after Radical Prostatectomy," Urology, Vol. 51, No. 6, 1998, pp. 970-976. doi:10.1016/S0090-4295(98)00103-4

[27] G. S. Bova, A. W. Partin, S. D. Isaacs, et al., "Biological Aggressiveness of Hereditary Prostate Cancer: Long-Term
Evaluation Following Radical Prostatectomy," The Journal of urology, Vol. 160, No. 3, 1998, pp. 660-663. doi:10.1016/S0022-5347(01)62748-4

[28] O. Bratt, U. Kristoffersson, R. Lundgren and H. Olsson, "Familial and Hereditary Prostate Cancer in Southern Sweden. A Population-Based Case-Control Study," European Journal of Cancer, Vol. 35, No. 2, 1999, pp. 272-277.

[29] T. M. King, L. Tong, R. J. Pack, C. Spencer and C. I. Amos, "Accuracy of Family History of Cancer as Reported by Men with Prostate Cancer," Urology, Vol. 59, No. 4, 2002, pp. 546-550. doi:10.1016/S0090-4295(01)01598-9

[30] G. H. Rauscher and D. P. Sandler, "Validating Cancer Histories in Deceased Relatives," Epidemiology, Vol. 16, No. 2, 2005, pp. 262-265. 